\title{
Dynamic Compensator-Based Output Feedback Controller Design for Uncertain Systems with Adjustable Robustness
}

\author{
Jeang-Lin Chang $(D)$ and Tsui-Chou Wu \\ Department of Electrical Engineering, Oriental Institute of Technology, No. 58, Section 2, Sihchuan Road, \\ Ban-Chiao, New Taipei City 220, Taiwan \\ Correspondence should be addressed to Jeang-Lin Chang; fe035@mail.oit.edu.tw
}

Received 11 April 2017; Revised 18 August 2017; Accepted 17 December 2017; Published 22 January 2018

Academic Editor: Juan-Albino Méndez-Pérez

Copyright (C) 2018 Jeang-Lin Chang and Tsui-Chou Wu. This is an open access article distributed under the Creative Commons Attribution License, which permits unrestricted use, distribution, and reproduction in any medium, provided the original work is properly cited.

\begin{abstract}
A low-order dynamics compensator-based output feedback stabilization method for a class of uncertain linear MIMO systems with mismatched disturbances is presented. Since all the system states are not measurable, the proposed controller inherently has low-pass filter property in which it can successfully replace the derivative terms of the system output and hence effectively estimate the input disturbance. The control scheme proposed here can simultaneously consider the input saturation problem and obtain the desired performance. Although the system has unknown uncertainties and disturbances, the uniformly ultimate boundedness of system states in the closed-loop system is analytically shown using the Lyapunov method. Finally, two numerical examples are presented to demonstrate the applicability of the proposed scheme.
\end{abstract}

\section{Introduction}

Control systems are always subject to unknown inputs in the form of internal uncertainties and exogenous disturbances that cannot be measured or pose inconvenience when making measurements. When not properly treated, the unknown inputs may result in poor performance of the control system. To attenuate the negative impact from unknown inputs and stabilize the closed-loop system, various methods including robust control [1-4] and sliding mode control [5-10] have been proposed. Moreover, in most practical systems, not all the system states are available. Previous research [5-10] has designed output feedback controllers using the sliding mode technique to stabilize multivariable plants, while the high-gain control is employed to guarantee that the system can reach and slide on the sliding surface in finite time. The high-gain control design suffers from the drawback of peaking phenomenon, in which the control input peaks to an extremely large value during the transient stage. The peaking phenomenon can easily violate the control saturation constraint and excite the unmodelled high-order dynamics. In order to fulfil the bounded input constraint while achieving robustness simultaneously, a control scheme for adjusting robustness during the transient time and for guaranteeing the robust performance in the steady state is presented in this paper.

There is another control method for attenuating the effect of unknown inputs in which an observer algorithm is first designed to estimate the unknown input and then cancel its effect by the control input. There are various formulations related to the unknown input estimation [11-20]. The disturbance observer is known to be very effective in eliminating disturbances [11, 12] and is very popular for robust motion control $[13,14]$. The main problem with this approach is that it is difficult for implementation in MIMO systems. Another approach to disturbance estimation involves designing a joint state and disturbance observer $[15,16]$ that estimates simultaneously the states and the unknown disturbances. This approach was first developed in studies of unknown input observers $[17,18]$. However, these previous works did not consider the issue of structure uncertainties. Moreover, one study [19] pointed out theoretically and experimentally that disturbance observer-based controllers may not be robust to large mismatched model uncertainty.

This research develops a dynamic compensator-based output feedback controller with guaranteed robust stability 
for linear MIMO systems with norm-bounded uncertainties and unknown disturbances. It should be noted that the maximum control effort usually occurs during the transient period. To simultaneously consider input constraint and obtain desired performance, an adjusted robustness, developed in this paper, can be ensured during the transient period while a robust performance can be guaranteed in the steady state. Using this scheme can limit the control effort within a predefined region if certain tolerance of transient response is permitted. Robust stability of the closed-loop system is guaranteed because the algorithm proposed by Gadewadikar et al. [1] is capable of obtaining the solution to the static output feedback problem. The proposed control law inherently has low-pass filter property that can successfully replace the derivative term of the system output, thus effectively eliminating the effect of input disturbance when the control gain is high enough. Without introducing any observer into the controller, it is shown that the system states are finally constrained in a small bounded region using the Lyapunov method. The method developed here is a low-order dynamic output feedback controller that is simple and easy to implement. Compared with other output feedback sliding mode controllers [5-10], the proposed control law, which involves only original system matrix parameters and does not need the discontinuous switching term, can obtain similar control results. Although the dynamic output feedback controller raises issues of control complexity, the performance of attenuating disturbance in the proposed method is evident.

This paper is organized as follows. The problem formulation of output feedback stabilization of uncertain linear MIMO systems is presented in Section 2. A dynamic compensator-based output feedback controller with guaranteeing robust stability of the closed-loop system and obtaining the desired performance is shown in Section 3. In Section 4, the system of the L-1011 aircraft at certain flight conditions is adopted to demonstrate the effectiveness of the proposed method. Moreover, we give simulation results comparing with the output feedback sliding mode controllers. Finally, the conclusions are given in Section 5.

\section{Problem Formulation}

Consider the following uncertain linear MIMO system:

$$
\begin{aligned}
& \dot{\mathbf{x}}(t)=\mathbf{A} \mathbf{x}(t)+\mathbf{B u}(t)+\mathbf{f}(\mathbf{x}, t) \\
& \mathbf{y}(t)=\mathbf{C} \mathbf{x}(t),
\end{aligned}
$$

where $\mathbf{x} \in \mathfrak{R}^{n}, \mathbf{u} \in \mathfrak{R}^{m}$, and $\mathbf{y} \in \mathfrak{R}^{l}$ are system states, control inputs, and system outputs, respectively. The function f $\in \mathfrak{R}^{n}$ is a time-varying vector which represents the lump sum of disturbances and/or uncertainties. Suppose that system matrices $\mathbf{A}, \mathbf{B}$, and $\mathbf{C}$ are known and have appropriate dimensions. The function $\mathbf{f}(\mathbf{x}, t)$ including the unknown disturbance and system uncertainty satisfies the following condition:

$$
\mathbf{f}(\mathbf{x}, t)=\mathbf{E d}(t)+\mathbf{D G}(t) \mathbf{H} \mathbf{x}(t),
$$

where $\mathbf{d} \in \mathfrak{R}^{p}$ is the unknown disturbance and $\mathbf{D}$ and $\mathbf{H}$ are known matrices with appropriate dimensions. The matrix $\mathbf{G}(t)$, being sufficiently smooth, is an unknown matrix satisfying $\mathbf{G}^{T}(t) \mathbf{G}(t) \leq \mathbf{I}$. According to (2), system (1) becomes

$$
\begin{aligned}
& \dot{\mathbf{x}}(t)=(\mathbf{A}+\mathbf{D G}(t) \mathbf{H}) \mathbf{x}(t)+\mathbf{B u}(t)+\mathbf{E} \mathbf{d}(t) \\
& \mathbf{y}(t)=\mathbf{C} \mathbf{x}(t)
\end{aligned}
$$

For system (3), the main goal is to design an output feedback controller such that the states in the presence of unknown uncertainties and disturbances are bounded in a small region. Because the disturbance is unknown and only the system output is measurable, many papers [2-10] apply the highgain control technique to obtain the desired performance. However, the high-gain control usually suffers from the input saturation and excites high frequency unmodelled dynamics. A design procedure which can fulfil the bounded input constraint and achieve robustness of the closed-loop system is presented in this paper. The proposed control scheme can effectively eliminate input disturbance and guarantee robust stability of the closed-loop system. As a result, the problem of peaking arising in conventional disturbance rejection controllers [2-10] can be effectively avoided using the control algorithm proposed here. Before introducing the proposed method, the following three assumptions are made throughout this paper. Nevertheless, it is in this respect that these three assumptions grow out of the output feedback controller and/or unknown input observer design methods [2-10, 15-18].

Assumption 1. The pairs (A, B) and (A, C) are stabilizable and detectable, respectively.

Assumption 2. Matrices B, E, and C have full rank, and $\operatorname{rank}(\mathbf{C E})=\operatorname{rank}(\mathbf{E})=p$.

Assumption 3. The triple (C, A, B) is minimum phase.

\section{Dynamic Output Feedback Controller Design}

This section constructs a dynamic output feedback control law, which can effectively eliminate the equivalent input disturbance and guarantee robust stability of the closedloop system. In comparison with the other output feedback sliding mode controllers [5-10], the proposed control law can effectively compensate for the effect of input disturbance and avoid the high-gain phenomenon in the transient time.

Let $\bullet^{+}$denote the pseudoinverse matrix of $\bullet$. Since Assumption 2 holds, the low-order dynamic compensatorbased output feedback controller for system (3) is designed as

$$
\begin{aligned}
\mathbf{u}(t)= & -\mathbf{K y}(t)-\beta \mathbf{B}^{+} \mathbf{E}\left((\mathbf{C E})^{+} \mathbf{y}(t)-\boldsymbol{\eta}(t)\right) \\
\dot{\boldsymbol{\eta}}(t) & \\
= & \left(-(\mathbf{C E})^{+} \mathbf{C B}\left(\mathbf{K}+\beta \mathbf{B}^{+} \mathbf{E}(\mathbf{C E})^{+}\right)+\beta(\mathbf{C E})^{+}\right) \mathbf{y}(t) \\
& +\beta\left((\mathbf{C E})^{+} \mathbf{C B B}{ }^{+} \mathbf{E}-\mathbf{I}_{P}\right) \boldsymbol{\eta}(t),
\end{aligned}
$$


where $\boldsymbol{\eta} \in \mathfrak{R}^{p}$ and $\mathbf{K} \in \mathfrak{R}^{m \times l}$ are the compensator state vector and the gain matrix designed in the following, respectively. Moreover, the parameter $\beta>0$ is a design constant for adjusting the performance of input constraint and robustness. To analyze the closed-loop system behavior, a new variable is defined as $\mathbf{z}=\beta\left((\mathbf{C E})^{+} \mathbf{y}-\boldsymbol{\eta}\right)$ and the control input is rewritten as

$$
\begin{aligned}
& \mathbf{u}(t)=-\mathbf{K y}(t)-\mathbf{B}^{+} \mathbf{E z}(t) \\
& \dot{\boldsymbol{\eta}}(t)=-(\mathbf{C E})^{+} \mathbf{C B}\left(\mathbf{K y}(t)+\mathbf{B}^{+} \mathbf{E z}(t)\right)+\mathbf{z}(t) .
\end{aligned}
$$

Substituting the control input (5) into system (3) yields the closed-loop system dynamics as

$$
\begin{aligned}
\dot{\mathbf{x}}(t)= & (\mathbf{A}-\mathbf{B K C}+\mathbf{D G}(t) \mathbf{H}) \mathbf{x}(t)+\mathbf{E d}(t) \\
& -\mathbf{B B}^{+} \mathbf{E z}(t) .
\end{aligned}
$$

Take the time derivate of $\mathbf{z}$ to obtain its dynamic equation as

$$
\begin{aligned}
\dot{\mathbf{z}}(t) & =\beta\left((\mathbf{C E})^{+} \dot{\mathbf{y}}(t)-\dot{\boldsymbol{\eta}}(t)\right)=\beta\left((\mathbf{C E})^{+}\right. \\
& \cdot \mathbf{C}(\mathbf{A x}(t)+\mathbf{D G}(t) \mathbf{H} \mathbf{x}(t)+\mathbf{B u}(t)+\mathbf{E d}(t)) \\
+ & \left.(\mathbf{C E})^{+} \mathbf{C B}\left(\mathbf{K y}(t)+\mathbf{B}^{+} \mathbf{E z}(t)\right)-\mathbf{z}(t)\right)=\beta\left((\mathbf{C E})^{+}\right. \\
& \cdot \mathbf{C}(\mathbf{A}+\mathbf{D G}(t) \mathbf{H}) \mathbf{x}(t)+\mathbf{d}(t)-\mathbf{z}(t)) .
\end{aligned}
$$

Let $\varepsilon=1 / \beta>0$. Then the dynamic equation of $\mathbf{z}$ becomes

$$
\varepsilon \dot{\mathbf{z}}(t)=(\mathbf{C E})^{+} \mathbf{C}(\mathbf{A}+\mathbf{D G}(t) \mathbf{H}) \mathbf{x}(t)+\mathbf{d}(t)-\mathbf{z}(t) .
$$

Augmenting (6) and (8) in a matrix form yields

$$
\begin{aligned}
& {\left[\begin{array}{c}
\dot{\mathbf{x}}(t) \\
\varepsilon \dot{\mathbf{z}}(t)
\end{array}\right]} \\
& =\left[\begin{array}{cc}
(\mathbf{A}+\mathbf{D G}(t) \mathbf{H}-\mathbf{B K C}) & -\mathbf{B B}^{+} \mathbf{E} \\
(\mathbf{C E})^{+} \mathbf{C}(\mathbf{A}+\mathbf{D G}(t) \mathbf{H}) & -\mathbf{I}_{p}
\end{array}\right]\left[\begin{array}{l}
\mathbf{x}(t) \\
\mathbf{z}(t)
\end{array}\right] \\
& \quad+\left[\begin{array}{c}
\mathbf{E} \\
\mathbf{I}_{p}
\end{array}\right] \mathbf{d}(t) .
\end{aligned}
$$

It is clear that system (9) is the standard form of a singular perturbation [21-23]. According to the classical theory of singular perturbation $[21,22]$, we can decompose system (8) into fast and slow subsystems. The slow reduced subsystem is found by making $\varepsilon=0$ in (8), thus yielding

$$
\mathbf{z}_{s}(t)=(\mathbf{C E})^{+} \mathbf{C}(\mathbf{A}+\mathbf{D G}(t) \mathbf{H}) \mathbf{x}_{s}(t)+\mathbf{d}(t),
$$

where $\mathbf{x}_{s}, \mathbf{z}_{s}$, and $\mathbf{y}_{s}$ denote the slow portions of the original variables $\mathbf{x}, \mathbf{z}$, and $\mathbf{y}$, respectively. She et al. [14] called this term in (10) input disturbance. As a result, the proposed method can effectively estimate input disturbance when the control gain $\beta$ is high enough. Let $\mathbf{A}_{1}=\left(\mathbf{I}_{n}-\mathbf{B B} \mathbf{B}^{+} \mathbf{E}(\mathbf{C E})^{+} \mathbf{C}\right) \mathbf{A}$ and $\mathbf{D}_{1}=\left(\mathbf{I}_{n}-\mathbf{B B}^{+} \mathbf{E}(\mathbf{C E})^{+} \mathbf{C}\right) \mathbf{D}$. Substituting (10) into system (6) gives

$$
\begin{aligned}
\dot{\mathbf{x}}_{s}(t)= & (\mathbf{A}-\mathbf{B K C}+\mathbf{D G}(t) \mathbf{H}) \mathbf{x}_{s}(t) \\
& -\mathbf{B B}^{+} \mathbf{E}(\mathbf{C E})^{+} \mathbf{C}(\mathbf{A}+\mathbf{D G}(t) \mathbf{H}) \mathbf{x}_{s}(t) \\
& +\left(\mathbf{I}_{n}-\mathbf{B} \mathbf{B}^{+}\right) \mathbf{E d}(t) \\
= & \left(\mathbf{I}_{n}-\mathbf{B} \mathbf{B}^{+} \mathbf{E}(\mathbf{C E})^{+} \mathbf{C}\right)(\mathbf{A}+\mathbf{D G}(t) \mathbf{H}) \mathbf{x}_{s}(t) \\
& -\mathbf{B K \mathbf { y } _ { s }}(t)+\left(\mathbf{I}_{n}-\mathbf{B} \mathbf{B}^{+}\right) \mathbf{E d}(t) \\
= & \left(\mathbf{A}_{1}+\mathbf{D}_{1} \mathbf{G}(t) \mathbf{H}-\mathbf{B K C}\right) \mathbf{x}_{s}(t) \\
& +\left(\mathbf{I}_{n}-\mathbf{B} \mathbf{B}^{+}\right) \mathbf{E d}(t) .
\end{aligned}
$$

System (11) is called the slow reduced system [23]. Moreover, the term $-\mathbf{K y}_{s}$ in system (11) can be taken as the control input and be designed to stabilize the matrix $\mathbf{A}_{1}+\mathbf{D}_{1} \mathbf{G}(t) \mathbf{H}$ using the static output feedback control technique. Let

$$
\mathbf{w}(t)=\mathbf{z}(t)-(\mathbf{C E})^{+} \mathbf{C}(\mathbf{A}+\mathbf{D G}(t) \mathbf{H}) \mathbf{x}(t)-\mathbf{d}(t),
$$

and then we from (6) obtain

$$
\begin{aligned}
\dot{\mathbf{x}}(t) & \\
& =\left(\left(\mathbf{I}_{n}-\mathbf{B B}^{+} \mathbf{E}(\mathbf{C E})^{+} \mathbf{C}\right)(\mathbf{A}+\mathbf{D G}(t) \mathbf{H})-\mathbf{B K C}\right) \\
& \cdot \mathbf{x}(t)-\mathbf{B w}(t)+\left(\mathbf{I}_{n}-\mathbf{B B}^{+}\right) \mathbf{E d}(t) \\
& =\left(\mathbf{A}_{1}+\mathbf{D}_{1} \mathbf{G}(t) \mathbf{H}-\mathbf{B K C}\right)-\mathbf{B} \mathbf{w}(t)+\left(\mathbf{I}_{n}-\mathbf{B B}^{+}\right) \\
& \cdot \mathbf{E d}(t) .
\end{aligned}
$$

From (8), the dynamic equation of $\mathbf{w}$ can be obtained as

$$
\begin{aligned}
\varepsilon \frac{d \mathbf{w}(t)}{d t} & \\
= & \left((\mathbf{C E})^{+} \mathbf{C}(\mathbf{A}+\mathbf{D G}(t) \mathbf{H}) \mathbf{x}(t)+\mathbf{d}(t)\right)-\mathbf{z}(t) \\
& \quad+\varepsilon \frac{d}{d t}\left((\mathbf{C E})^{+} \mathbf{C}(\mathbf{A}+\mathbf{D G}(t) \mathbf{H}) \mathbf{x}(t)+\mathbf{d}(t)\right) \\
= & -\mathbf{w}(t)+\varepsilon \mathbf{q}(t),
\end{aligned}
$$

where $\mathbf{q}(t)=(d / d t)\left((\mathbf{C E})^{+} \mathbf{C}(\mathbf{A}+\mathbf{D G}(t) \mathbf{H}) \mathbf{x}(t)+\mathbf{d}(t)\right)$. Let $\mathbf{x}(t, \varepsilon)$ be the solution of the closed-loop system (9). Then the system performance satisfies the following theorem.

Lemma 4. If the matrix $\mathbf{B}$ is full rank, then $\left\|\left(\mathbf{I}_{n}-\mathbf{B B}^{+}\right) \mathbf{E}\right\| \leq$ $\|\mathbf{E}\|$ where the operator $\|\bullet\|$ denotes the 2-norm of the matrix $\bullet$.

Proof. Since $\mathbf{B}^{+}$is the pseudoinverse of $\mathbf{B}, \mathbf{B}^{+}=\left(\mathbf{B}^{T} \mathbf{B}\right)^{-1} \mathbf{B}^{T}$. Note that

$$
\begin{aligned}
\left(\mathbf{I}_{n}\right. & \left.-\mathbf{B B}^{+}\right)^{T}\left(\mathbf{I}_{n}-\mathbf{B B}^{+}\right) \\
& =\left(\mathbf{I}_{n}-\mathbf{B}\left(\mathbf{B}^{T} \mathbf{B}\right)^{-1} \mathbf{B}^{T}\right)\left(\mathbf{I}_{n}-\mathbf{B}\left(\mathbf{B}^{T} \mathbf{B}\right)^{-1} \mathbf{B}^{T}\right) \\
& =\mathbf{I}_{n}-\mathbf{B}\left(\mathbf{B}^{T} \mathbf{B}\right)^{-1} \mathbf{B}^{T}=\mathbf{I}_{n}-\mathbf{B} \mathbf{B}^{+} .
\end{aligned}
$$


Suppose $\mathbf{v} \in \mathfrak{R}^{n}$ is an eigenvector of $\mathbf{I}_{n}-\mathbf{B B}^{+}$associated with the eigenvalue $\lambda \in \mathfrak{R}$. From

$$
\begin{gathered}
\mathbf{v}^{T}\left(\mathbf{I}_{n}-\mathbf{B B}^{+}\right)^{T}\left(\mathbf{I}_{n}-\mathbf{B B}^{+}\right) \mathbf{v}=\lambda^{2}\|\mathbf{v}\|^{2} \\
=\mathbf{v}^{T}\left(\mathbf{I}_{n}-\mathbf{B B}^{+}\right) \mathbf{v}=\lambda\|\mathbf{v}\|^{2},
\end{gathered}
$$

we have $\lambda^{2}=\lambda$ and hence the eigenvalues of $\mathbf{I}_{n}-\mathbf{B} \mathbf{B}^{+}$obtained $\lambda=0$ or $\lambda=1$. According to the definition of the 2-norm, we have $\left\|\mathbf{I}_{n}-\mathbf{B B}^{+}\right\|=1$ and

$$
\left\|\left(\mathbf{I}_{n}-\mathbf{B B}^{+}\right) \mathbf{E}\right\| \leq\left\|\mathbf{I}_{n}-\mathbf{B B}^{+}\right\|\|\mathbf{E}\| \leq\|\mathbf{E}\| .
$$

The proof of this lemma is finished.

Theorem 5. Consider system (1) with Assumptions 1-3 satisfied and design the controller as (4). If the following conditions are satisfied:

(1) the gain matrix $\mathbf{K}$ can be designed to stabilize $\mathbf{A}_{1}+$ $\mathbf{D}_{1} \mathbf{G}(t) \mathbf{H}-\mathbf{B K C}$,

(2) the function $\mathbf{f}(\mathbf{x}, t)$ and its first partial derivative with respect to $(\mathbf{x}, t)$ are continuous and globally bounded,

then there exists a positive constant $\varepsilon^{*}>0$ such that, for all $0<\varepsilon<\varepsilon^{*}$, the solution $\mathbf{x}(t, \varepsilon)$ has the following property:

$$
\mathbf{x}(t, \varepsilon)-\overline{\mathbf{x}}(t)=O(\varepsilon)
$$

where $\overline{\mathbf{x}}(t)$ is the solution of the reduced system

$$
\begin{aligned}
\dot{\mathbf{x}}(t)= & \left(\mathbf{A}_{1}+\mathbf{D}_{1} \mathbf{G}(t) \mathbf{H}-\mathbf{B K C}\right) \mathbf{x}(t) \\
& +\left(\mathbf{I}_{n}-\mathbf{B B}^{+}\right) \mathbf{E d}(t) .
\end{aligned}
$$

Proof. Since Assumption 3 holds and $\mathbf{G}(t)$ is sufficiently smooth, according to time-scale properties of singular perturbation [23], the boundary layer system can be obtained as

$$
\frac{d \mathbf{w}(\tau)}{d \tau}=-\mathbf{w}(\tau)
$$

where the new time scale $\tau$ is related to the original time $t$ via the relationship $\tau=t / \varepsilon$. System (20) is called the fast system [23]. It follows from (20) that the boundary layer system is exponentially stable. Since the matrix $\mathbf{A}_{1}+\mathbf{D}_{1} \mathbf{G}(t) \mathbf{H}-\mathbf{B K C}$ for the reduced system in (11) is stable and the boundary layer system (20) is exponentially stable, we can apply Tikhonov's theorem [23] to show that there exists a positive constant $\varepsilon^{*}>$ 0 such that, for all $0<\varepsilon<\varepsilon^{*}$, the solution $\mathbf{x}(t, \varepsilon)$ has the following property:

$$
\mathbf{x}(t, \varepsilon)-\overline{\mathbf{x}}(t)=O(\varepsilon) .
$$

The proof of the theorem is finished.

Since $\left\|\left(\mathbf{I}_{n}-\mathbf{B B}^{+}\right) \mathbf{E}\right\| \leq\|\mathbf{E}\|$, Theorem 5 and Lemma 4 indicate that if $\varepsilon \rightarrow 0$, that is, $\beta \rightarrow \infty$, the proposed control algorithm can obtain better disturbance rejection. It follows that increment of the parameter $\beta$ will definitely suppress the effect caused by system uncertainties and disturbances. However, this approach causes the high-gain control problem, resulting in the peaking phenomenon during the transient state. The peaking phenomenon can easily violate the control saturation constraint and excite the unmodelled high-order dynamics. On the other hand, decreasing $\beta$ will yield the poor performance but the maximal control effort can be reduced, thus achieving a physical limit of control input. Alternatively, the weighting factor $\beta$ will be determined by a tradeoff between input constraint and robust performance. This scheme can achieve an adjusted robustness during the transient period and robust performance in the steady state. In the following, the static output feedback technique, proposed by Gadewadikar et al. [1], is employed to design the gain $\mathbf{K}$ and analyze robust stability of the closed-loop system.

It follows from (6) and (7) that the closed-loop system can be written as

$$
\begin{aligned}
& {\left[\begin{array}{c}
\dot{\mathbf{x}}(t) \\
\dot{\mathbf{z}}(t)
\end{array}\right]} \\
& =\left[\begin{array}{cc}
(\mathbf{A}-\mathbf{B K C}+\mathbf{D G}(t) \mathbf{H}) & -\mathbf{B B}^{+} \mathbf{E} \\
\beta(\mathbf{C E})^{+} \mathbf{C}(\mathbf{A}+\mathbf{D G}(t) \mathbf{H}) & -\beta \mathbf{I}_{p}
\end{array}\right]\left[\begin{array}{l}
\mathbf{x}(t) \\
\mathbf{z}(t)
\end{array}\right] \mathbf{x}(t) \\
& +\left[\begin{array}{r}
\mathbf{E} \\
\beta \mathbf{I}_{p}
\end{array}\right] \mathbf{d}(t) .
\end{aligned}
$$

From

$$
\begin{aligned}
& {\left[\begin{array}{cc}
(\mathbf{A}-\mathbf{B K C}+\mathbf{D G}(t) \mathbf{H}) & -\mathbf{B B}^{+} \mathbf{E} \\
\beta(\mathbf{C E})^{+} \mathbf{C}(\mathbf{A}+\mathbf{D G}(t) \mathbf{H}) & -\beta \mathbf{I}_{p}
\end{array}\right]} \\
& =\left[\begin{array}{cc}
\mathbf{A} & -\mathbf{B B}^{+} \mathbf{E} \\
\beta(\mathbf{C E})^{+} \mathbf{C A} & -\beta \mathbf{I}_{p}
\end{array}\right] \\
& \quad+\left[\begin{array}{c}
\mathbf{D} \\
\beta(\mathbf{C E})^{+} \mathbf{C D}
\end{array}\right] \mathbf{G}(t)\left[\begin{array}{ll}
\mathbf{H} & \mathbf{0}
\end{array}\right]-\left[\begin{array}{l}
\mathbf{B} \\
\mathbf{0}
\end{array}\right] \mathbf{K}\left[\begin{array}{ll}
\mathbf{C} & \mathbf{0}
\end{array}\right],
\end{aligned}
$$

we know that (22) becomes

$$
\begin{aligned}
{\left[\begin{array}{c}
\dot{\mathbf{x}}(t) \\
\dot{\mathbf{z}}(t)
\end{array}\right]=} & (\overline{\mathbf{A}}+\overline{\mathbf{D}} \mathbf{G}(t) \overline{\mathbf{H}}-\overline{\mathbf{B}} \mathbf{K} \overline{\mathbf{C}})\left[\begin{array}{l}
\mathbf{x}(t) \\
\mathbf{z}(t)
\end{array}\right] \mathbf{x}(t) \\
& +\overline{\mathbf{E}} \mathbf{d}(t),
\end{aligned}
$$

where $\overline{\mathbf{A}}=\left[\begin{array}{cc}\mathbf{A} & -\mathbf{B B}^{+} \mathbf{E} \\ \beta(\mathbf{C E})^{+} \mathbf{C A} & -\beta \mathbf{I}_{p}\end{array}\right] \in \mathfrak{R}^{(n+p) \times(n+p)}, \overline{\mathbf{B}}=\left[\begin{array}{l}\mathbf{B} \\ \mathbf{0}\end{array}\right] \epsilon$ $\mathfrak{R}^{(n+p) \times m}, \overline{\mathbf{E}}=\left[\begin{array}{c}\mathbf{E} \\ \beta \mathbf{I}_{p}\end{array}\right] \in \mathfrak{R}^{(n+p) \times p}, \overline{\mathbf{C}}=\left[\begin{array}{ll}\mathbf{C} & \mathbf{0}\end{array}\right] \in \mathfrak{R}^{l \times(n+p)}$, $\overline{\mathbf{D}}=\left[\begin{array}{c}\mathbf{D} \\ \beta(\mathbf{C E})^{+} \mathbf{C D}\end{array}\right]$, and $\overline{\mathbf{H}}=\left[\begin{array}{ll}\mathbf{H} & \mathbf{0}\end{array}\right]$. Let the symbols $\lambda_{\min }(\bullet)$ and $\lambda_{\text {max }}(\bullet)$ denote the minimum and maximum eigenvalues of the matrix $\bullet$, respectively. Since Assumption 1 holds, it is easy to check that the pairs $(\overline{\mathbf{A}}, \overline{\mathbf{B}})$ and $(\overline{\mathbf{C}}, \overline{\mathbf{A}})$ are stabilizable and detectable, respectively. Then we have the following theorem.

Theorem 6. Consider system (1) with Assumptions 1-3 satisfied and design the control input as (4). Let $\mathbf{R}>0$ and $\mathbf{Q}>0$ be the weighting matrices, and let $\eta>0$ be a constant. If the gain $\mathbf{K}$ and the parameter matrix $\mathbf{L}$ are designed to satisfy the condition

$$
\mathbf{K} \overline{\mathbf{C}}=\mathbf{R}^{-1}\left(\overline{\mathbf{B}}^{T} \mathbf{P}+\mathbf{L}\right)
$$


where matrices $\mathbf{L}$ and $\mathbf{P}>0$ satisfy the following algebraic Riccati equation

$$
\begin{gathered}
\overline{\mathbf{A}}^{T} \mathbf{P}+\mathbf{P} \overline{\mathbf{A}}-\mathbf{P} \overline{\mathbf{B}} \mathbf{R}^{-1} \overline{\mathbf{B}}^{T} \mathbf{P}+\mathbf{L}^{T} \mathbf{R}^{-1} \mathbf{L}+\frac{1}{\eta} \mathbf{P} \overline{\mathbf{D}} \overline{\mathbf{D}}^{T} \mathbf{P} \\
+\eta \overline{\mathbf{H}}^{T} \overline{\mathbf{H}}+\mathbf{Q}=\mathbf{0},
\end{gathered}
$$

then the system performance satisfies the following property:

$$
\|\mathbf{x}(t)\| \leq \frac{2\|\mathbf{P} \overline{\mathbf{E}}\| \bar{d}}{\rho \lambda_{\text {min }}\left(\mathbf{Q}+\overline{\mathbf{C}}^{T} \mathbf{K}^{T} \mathbf{R} \mathbf{K} \overline{\mathbf{C}}\right)} \sqrt{\frac{\lambda_{\text {max }}(\mathbf{P})}{\lambda_{\text {min }}(\mathbf{P})}},
$$

where $\|\mathbf{d}\| \leq \bar{d}$ and $0<\rho<1$ is a constant.

Proof. Substitute the relation in (26) into (24) to obtain the closed-loop system dynamics as

$$
\begin{aligned}
\dot{\mathbf{x}}(t)= & \left(\overline{\mathbf{A}}-\overline{\mathbf{B}} \mathbf{R}^{-1}\left(\overline{\mathbf{B}}^{T} \mathbf{P}+\mathbf{L}\right)+\overline{\mathbf{D}} \mathbf{G}(t) \overline{\mathbf{H}}\right) \mathbf{x}(t) \\
& +\overline{\mathbf{E}} \mathbf{d}(t)=\left(\mathbf{A}_{s}+\overline{\mathbf{D}} \mathbf{G}(t) \overline{\mathbf{H}}\right) \mathbf{x}(t)+\overline{\mathbf{E}} \mathbf{d}(t),
\end{aligned}
$$

where $\mathbf{A}_{s}=\overline{\mathbf{A}}-\overline{\mathbf{B}} \mathbf{R}^{-1}\left(\overline{\mathbf{B}}^{T} \mathbf{P}+\mathbf{L}\right)$. First, we show that the matrix $\overline{\mathbf{A}}+\overline{\mathbf{D}} \mathbf{G}(t) \overline{\mathbf{H}}-\overline{\mathbf{B}} \mathbf{R}^{-1}\left(\overline{\mathbf{B}}^{T} \mathbf{P}+\mathbf{L}\right)$ is asymptotically stable. Rearranging the algebraic Riccati equation (26) yields

$$
\begin{gathered}
\mathbf{A}_{s}^{T} \mathbf{P}+\mathbf{P A}_{s}+\frac{1}{\eta} \mathbf{P} \overline{\mathbf{D}} \overline{\mathbf{D}}^{T} \mathbf{P}+\eta \overline{\mathbf{H}}^{T} \overline{\mathbf{H}} \\
=-\mathbf{Q}-\overline{\mathbf{C}}^{T} \mathbf{K}^{T} \mathbf{R} \mathbf{K} \overline{\mathbf{C}}<0 .
\end{gathered}
$$

Choose a Lyapunov function $V(t)=\mathbf{x}^{T}(t) \mathbf{P} \mathbf{x}(t)$ where the positive definite matrix $\mathbf{P}$ satisfies (26). Taking the time derivative of $V(t)$ obtains

$$
\begin{aligned}
& \dot{V}(t)=-\mathbf{x}^{T}(t) \\
& \cdot\left(\left(\mathbf{A}_{s}+\overline{\mathbf{D}} \mathbf{G}(t) \overline{\mathbf{H}}\right)^{T} \mathbf{P}+\mathbf{P}\left(\mathbf{A}_{s}+\overline{\mathbf{D}} \mathbf{G}(t) \overline{\mathbf{H}}\right)\right) \mathbf{x}(t) \\
& +2 \mathbf{x}^{T}(t) \mathbf{P} \overline{\mathbf{E}} \mathbf{d}(t) .
\end{aligned}
$$

Since $\mathbf{G}^{T}(t) \mathbf{G}(t) \leq \mathbf{I}$, we have

$$
(\overline{\mathbf{D}} \mathbf{G}(t) \overline{\mathbf{H}})^{T} \mathbf{P}+\mathbf{P} \overline{\mathbf{D}} \mathbf{G}(t) \overline{\mathbf{H}} \leq \eta \mathbf{P} \overline{\mathbf{D}} \overline{\mathbf{D}}^{T} \mathbf{P}_{x}+\frac{1}{\eta} \overline{\mathbf{H}}^{T} \overline{\mathbf{H}},
$$

where $\eta>0$ is a scalar. Applying the relation shown in (29) and substituting the above inequality into (30), we can obtain

$$
\begin{aligned}
& \dot{V}(t)=-\mathbf{x}^{T}(t) \\
& \cdot\left(\mathbf{Q}+\overline{\mathbf{C}}^{T} \mathbf{K}^{T} \mathbf{R} \mathbf{K} \overline{\mathbf{C}}+\frac{1}{\eta} \mathbf{P} \overline{\mathbf{D}} \overline{\mathbf{D}}^{T} \mathbf{P}+\eta \overline{\mathbf{H}}^{T} \overline{\mathbf{H}}\right) \mathbf{x}(t) \\
& +2 \mathbf{x}^{T}(t) \mathbf{P} \overline{\mathbf{D}} \mathbf{G}(t) \overline{\mathbf{H}} \mathbf{x}(t)+2 \mathbf{x}^{T}(t) \mathbf{P} \overline{\mathbf{E}} \mathbf{d}(t) \\
& \leq-\mathbf{x}^{T}(t)\left(\mathbf{Q}+\overline{\mathbf{C}}^{T} \mathbf{K}^{T} \mathbf{R} \mathbf{K} \overline{\mathbf{C}}\right) \mathbf{x}(t)+2 \mathbf{x}^{T}(t) \mathbf{P} \overline{\mathbf{E}} \mathbf{d}(t) \\
& \leq-\lambda_{\min }\left(\mathbf{Q}+\overline{\mathbf{C}}^{T} \mathbf{K}^{T} \mathbf{R} \mathbf{K} \overline{\mathbf{C}}\right)\|\mathbf{x}(t)\|^{2}+2 \bar{d}\|\mathbf{P} \overline{\mathbf{E}}\| \\
& \cdot\|\mathbf{x}(t)\|=-(1-\rho) \lambda_{\min }(\mathbf{Y})\|\mathbf{x}(t)\|^{2}-\|\mathbf{x}(t)\| \\
& \cdot\left(\rho \lambda_{\min }(\mathbf{Y})\|\mathbf{x}(t)\|-2 \bar{d}\|\mathbf{P} \overline{\mathbf{E}}\|\right),
\end{aligned}
$$

where $0<\rho<1$ is a constant and $\mathbf{Y}=\mathbf{Q}+\overline{\mathbf{C}}^{T} \mathbf{K}^{T} \mathbf{R} \mathbf{K} \overline{\mathbf{C}}>0$. If $\|\mathbf{x}(t)\| \geq 2 \bar{d}\|\mathbf{P} \overline{\mathbf{E}}\| / \rho \lambda_{\text {min }}(\mathbf{Y})$, then the above equation becomes

$$
\dot{V}(t) \leq-(1-\rho) \lambda_{\min }(\mathbf{Y})\|\mathbf{x}(t)\|^{2}
$$

$$
\text { for }\|\mathbf{x}(t)\| \geq \frac{2 \bar{d}\|\mathbf{P} \overline{\mathbf{B}}\|}{\rho \lambda_{\min }(\mathbf{Y})} .
$$

Since $\lambda_{\min }(\mathbf{P})\|\mathbf{x}(t)\|^{2} \leq \mathbf{x}^{T}(t) \mathbf{P} \mathbf{x}(t) \leq \lambda_{\max }(\mathbf{P})\|\mathbf{x}(t)\|^{2}$, we can conclude from [23] that the system states are finally ultimately bounded

$$
\|\mathbf{x}(t)\| \leq \frac{2\|\mathbf{P} \overline{\mathbf{E}}\| \bar{d}}{\rho \lambda_{\min }\left(\mathbf{Q}+\overline{\mathbf{C}}^{T} \mathbf{K}^{T} \mathbf{R} \mathbf{K} \overline{\mathbf{C}}\right)} \sqrt{\frac{\lambda_{\max }(\mathbf{P})}{\lambda_{\min }(\mathbf{P})}} .
$$

Hence, the proof of the theorem is completed.

Both coupled equations (25) and (26) for static output feedback design to find the solution to $\mathbf{K}$ are generally complicated. Instead, Gadewadikar et al. [1] provided the iterative search algorithm to obtain the gain $\mathbf{K}$. The main advantage of their method is that the available well-developed techniques are employed to solve the algebraic Riccati equation (26). Since system (24) has the uncertain term affecting robust stability, the designer should carefully search these design parameters $\beta$, $\mathbf{Q}$, and $\mathbf{R}$, such that the solutions to (25) and (26) exist. Hence, the solution to $\mathbf{K}$ is obtained by trial and error.

Remark 7 . As $\beta \rightarrow \infty$, the proposed method can effectively compensate the effect of input disturbance. The resulting controller in (4) represents a high-gain controller. For practical control systems, tolerance is usually allowed due to certain system limitations, such as bandwidth and limited actuator ability. The allowable tolerance can be interpreted as the allowable robust performance of the system. Hence, the parameter $\beta$ cannot be too large for practical considering. According to the practical system specifications, the tradeoff parameter $\beta$ needs to be first chosen and then to obtain the system dynamic (24). Finally, we apply Gadewadikar's search algorithm [1] to the coupled equations (25) and (26) to find the solution to $\mathbf{K}$. 
Remark 8. More specifically, when the mismatched disturbance is defined in $L_{2}$-norm space, that is, $\mathbf{d} \in L_{2}$, we can use the property of robust disturbance attenuation to design the controller. The disturbance attenuation problem is to design an output feedback control law which can ensure that the effect of the disturbance acting on a system is reduced to an accepted level. Let $0 \leq \gamma<\infty$ and $\mathbf{R}>0$ be a design constant and the weighting matrix, respectively. For system (22), it is desired to find a static output feedback gain matrix $\mathbf{K}$ such that the closed-loop system is stable and its $L_{2}$ gain is bounded by a prescribed value $\gamma$. Similar to the work of Theorem 5 and Chang's paper [9], if the gain $\mathbf{K}$ and the parameter matrix $\mathbf{L}$ are designed to satisfy the condition

$$
\mathbf{K} \overline{\mathbf{C}}=-\mathbf{R}^{-1}\left(\mathbf{B}^{T} \mathbf{P}+\mathbf{L}\right)
$$

where the matrices $\mathbf{L}$ and $\mathbf{P}>0$ satisfy the algebraic Riccati equation

$$
\begin{aligned}
\overline{\mathbf{A}}^{T} \mathbf{P} & +\mathbf{P} \overline{\mathbf{A}}+\overline{\mathbf{C}}^{T} \overline{\mathbf{C}}+\frac{1}{\gamma^{2}} \mathbf{P} \overline{\mathbf{E}} \overline{\mathbf{E}}^{T} \mathbf{P}-\mathbf{P} \overline{\mathbf{B}} \mathbf{R}^{-1} \overline{\mathbf{B}}^{T} \mathbf{P} \\
& +\mathbf{L}^{T} \mathbf{R}^{-1} \mathbf{L}+\frac{1}{\beta} \mathbf{P} \overline{\mathbf{D}} \overline{\mathbf{D}}^{T} \mathbf{P}+\beta \overline{\mathbf{H}}^{T} \overline{\mathbf{H}}=\mathbf{0},
\end{aligned}
$$

then the matrix $\overline{\mathbf{A}}-\overline{\mathbf{B}} \mathbf{K} \overline{\mathbf{C}}=\overline{\mathbf{A}}-\overline{\mathbf{B}} \mathbf{R}^{-1}\left(\mathbf{B}^{T} \mathbf{P}+\mathbf{L}\right)$ is Hurwitz and the system performance satisfies

$$
\int_{0}^{t}\left(\mathbf{y}^{T} \mathbf{y}+\mathbf{v}^{T} \mathbf{R v}\right) d \tau \leq \gamma^{2} \int_{0}^{t}\left(\mathbf{d}^{T} \mathbf{d}\right) d \tau+\mathbf{x}(0)^{T} \mathbf{P x}(0)
$$

for $t \geq 0$,

where $\mathbf{v}(t)=-\overline{\mathbf{K}} \mathbf{C} \mathbf{x}(t)=-\mathbf{R}^{-1}\left(\mathbf{B}^{T} \mathbf{P}+\mathbf{L}\right) \mathbf{x}(t)$.

Remark 9. If the matrix $\mathbf{E}$ satisfies the so-called matched condition, it follows that there exists a matrix $\mathbf{R} \in \mathfrak{R}^{m \times p}$ such that $\mathbf{E}=\mathbf{B R}$. Then the slow reduced system in (11) becomes

$$
\dot{\mathbf{x}}_{s}(t)=\left(\mathbf{A}_{1}+\mathbf{D}_{1} \mathbf{G}(t) \mathbf{H}-\mathbf{B K C}\right) \mathbf{x}_{s}(t) .
$$

Its behavior is not affected by the matched perturbation. As seen in (38), the proposed control method is capable of eliminating the matched disturbance, thus obtaining the control effect similar to that when using a static output feedback sliding mode controller [4-9].

Remark 10. According to the proposed control scheme, we can from (5) and (7) rewrite the control input as

$$
\begin{aligned}
\mathbf{u}(t)= & -\mathbf{K y}(t)-\mathbf{B}^{+} \mathbf{E z}(t) \\
\dot{\mathbf{z}}(t)= & \beta(\mathbf{C E})^{+} \mathbf{C}(\mathbf{A}+\mathbf{D G}(t) \mathbf{H}) \mathbf{x}(t)+\beta \mathbf{d}(t) \\
& -\beta \mathbf{z}(t) .
\end{aligned}
$$

Since $(\mathbf{C E})^{+} \dot{\mathbf{y}}(t)=(\mathbf{C E})^{+} \mathbf{C}((\mathbf{A}+\mathbf{D G}(t) \mathbf{H}) \mathbf{x}(t)+\mathbf{B u}(t))+\mathbf{d}(t)$, it follows from the above equation that

$$
\begin{aligned}
\mathbf{u}(t) & =-\mathbf{K y}(t)-\frac{\beta}{(s+\beta)} \\
\cdot & \mathbf{B}^{+} \mathbf{E}\left((\mathbf{C E})^{+} \mathbf{C}(\mathbf{A}+\mathbf{D G}(t) \mathbf{H}) \mathbf{x}(t)+\mathbf{d}(t)\right) \\
& =-\mathbf{K y}(t)-\frac{\beta}{(s+\beta)} \\
& \cdot \mathbf{B}^{+} \mathbf{E}\left((\mathbf{C E})^{+} \dot{\mathbf{y}}(t)-(\mathbf{C E})^{+} \mathbf{C B u}(t)\right) .
\end{aligned}
$$

The control input (40) inherently has low-pass filter property and it can replace the velocity measurement. With the connection of an additional dynamic compensator, the proposed method can obtain the effect of desired differentiators and thus does not need any observer to estimate input disturbance.

\section{Numerical Examples}

Example 1. Consider the lateral axis dynamics of an L-1011 aircraft at certain flight conditions for demonstrating the developed control law in which its state space form is given by

$$
\dot{\mathbf{x}}(t)=\left[\begin{array}{cccc}
-2.98 & 0.93 & 0 & -0.0340 \\
-0.99 & -0.21 & 0.035 & -0.0011 \\
0 & 0 & 0 & 1 \\
0.39 & -5.555 & 0 & -1.89
\end{array}\right] \mathbf{x}(t)
$$

$$
+\left[\begin{array}{c}
-0.032 \\
0 \\
0 \\
-1.6
\end{array}\right] u(t)+\left[\begin{array}{c}
-0.032 \\
0.2 \\
0.1 \\
-1.6
\end{array}\right] d(t)
$$

$$
\mathbf{y}(t)=\left[\begin{array}{llll}
0 & 0 & 1 & 0 \\
0 & 0 & 0 & 1
\end{array}\right] \mathbf{x}(t)
$$

where the unknown disturbance is set as $d(t)=2 \sin (2 t)+$ $2 \cos (\pi t / 4)$. It is easy to check that the system satisfies three assumptions. Given $\mathbf{Q}=\mathbf{I}_{4}, \mathbf{R}=1$, and $\beta=5$ solving algebraic Riccati equations (25) and (26) gives

$$
\begin{aligned}
& \mathbf{P} \\
& =\left[\begin{array}{ccccc}
1.4284 & -3.6874 & 0.6707 & 0.1156 & -0.0086 \\
-3.6874 & 11.7797 & -2.1378 & -0.1847 & 0.2294 \\
0.6707 & -2.1378 & 1.9396 & 0.6264 & 0.1141 \\
0.1156 & -0.1847 & 0.6264 & 1.0206 & 0.3263 \\
-0.0086 & 0.2294 & 0.1141 & 0.3263 & 0.2070
\end{array}\right] .
\end{aligned}
$$




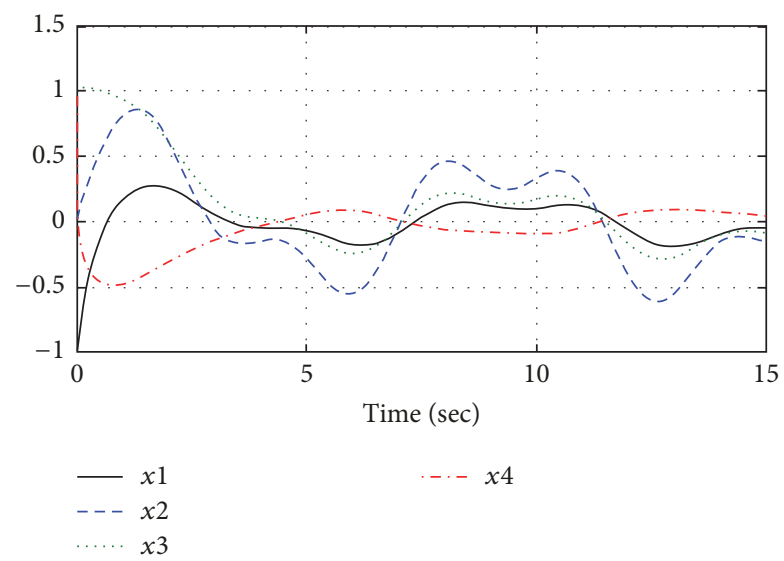

FIgURE 1: System states of the proposed method with $\beta=50$.

The controller is then designed as

$$
\begin{aligned}
u(t)= & {\left[\begin{array}{ll}
1.0237 & 1.6367
\end{array}\right] \mathbf{y}(t) } \\
& -5\left(\left[\begin{array}{ll}
0.1946 & -3.1128
\end{array}\right] \mathbf{y}(t)-\eta(t)\right) \\
\dot{\eta}(t)= & {\left[\begin{array}{ll}
1.0197 & 1.6303
\end{array}\right] \mathbf{y}(t)-0.0195 \eta(t) . }
\end{aligned}
$$

Figure 1 shows the response of the system states. It is clear that robust stability of the closed-loop system is guaranteed. For the sake of comparison, $\beta=50$ was introduced into the proposed method and the static output feedback $H_{\infty}$ control algorithm proposed by Gadewadikar et al. [1] is then employed to design the output feedback controller

$$
u(t)=\left[\begin{array}{ll}
3.2284 & 2.8654
\end{array}\right] \mathbf{y}(t) .
$$

Figures 2 and 3 illustrate the simulated responses of $\|\mathbf{y}(t)\|$ and the control input, respectively, for the three cases under the initial conditions $\mathbf{x}(0)=\left[\begin{array}{llll}-1 & 0 & 1 & 1\end{array}\right]^{T}$ in which Figures 2 and 3 show the responses of $\|\mathbf{y}(t)\|$ and the control input, respectively. As can be seen, the proposed method obtains good system performance when a bigger value of $\beta$ is used. The obvious advantages of the proposed control are that the peaking phenomenon of the control input is effectively avoided and the disturbance rejection performance is successfully improved. Hence, the parameter $\beta$ can be utilized to adjust system performance between input constraint and robustness. Although the compensator-based output feedback control law raises control complexity and incurs additional software, the proposed control scheme can achieve disturbance rejection and guarantee globally robust stability of the closed-loop system.

Example 2. To compare the developed control law with the other methods, a system proposed by Xiang et al. [6] is taken

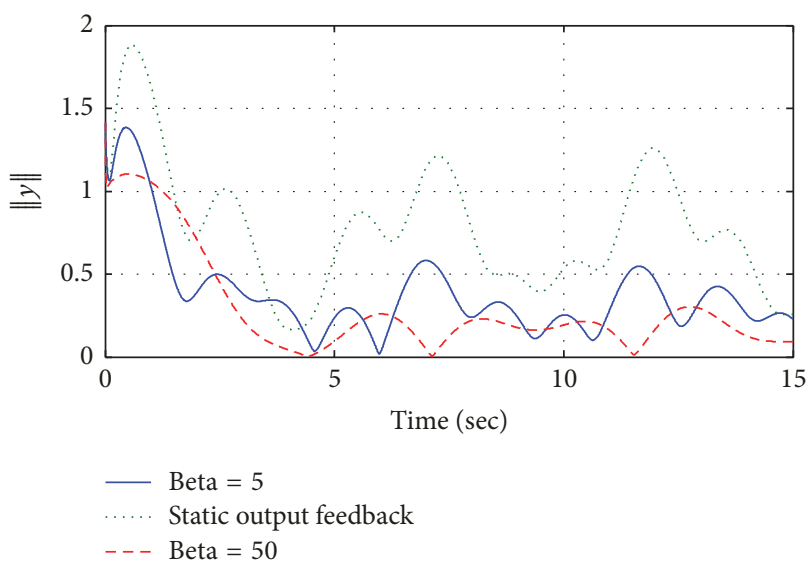

Figure 2: Responses of $\|\mathbf{y}(t)\|$ of the three cases.

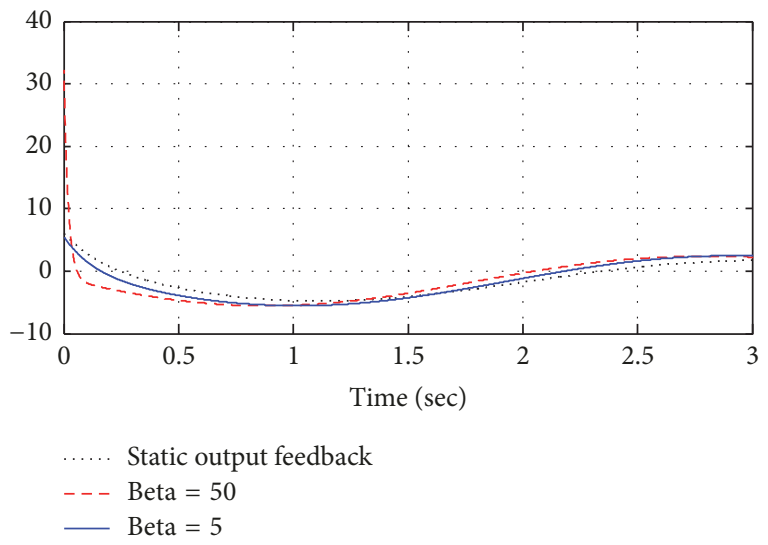

FIGURE 3: System input of the three cases.

as the following state space form:

$$
\begin{aligned}
\dot{\mathbf{x}}(t)= & {\left[\begin{array}{ccc}
-3+\sin (t) & 0 & 1+\sin (2 t) \\
1 & 2 & \sin (4 t) \\
0 & 1+\sin (3 t) & -2
\end{array}\right] \mathbf{x}(t) } \\
& +\left[\begin{array}{l}
0 \\
1 \\
0
\end{array}\right]\left(u(t)+2 \sin \left(\frac{\pi t}{4}\right)+2 \cos (2 t)\right) \\
\mathbf{y}(t)= & {\left[\begin{array}{lll}
0 & 1 & 0 \\
1 & 1 & 0
\end{array}\right] \mathbf{x}(t), }
\end{aligned}
$$

where the initial condition is altered as $\mathbf{x}(0)=\left[\begin{array}{lll}5 & 1 & -2\end{array}\right]^{T}$. Setting $\alpha=0.1, \beta=5, \mathbf{Q}=15 \mathbf{I}$, and $\mathbf{R}=0.1$ and then solving coupled equations (25) and (26) can yield the controller as

$$
\begin{aligned}
u(t)= & -\left[\begin{array}{ll}
21.6352 & -1.0958
\end{array}\right] \mathbf{y}(t) \\
& -5\left(\left[\begin{array}{ll}
0.5 & 0.5
\end{array}\right] \mathbf{y}(t)-\boldsymbol{\eta}(t)\right) \\
\dot{\boldsymbol{\eta}}(t)= & -\left[\begin{array}{ll}
21.6352 & -1.0958
\end{array}\right] \mathbf{y}(t) \\
& +0.1\left(\left[\begin{array}{ll}
0.5 & 0.5
\end{array}\right] \mathbf{y}(t)-\boldsymbol{\eta}(t)\right) .
\end{aligned}
$$




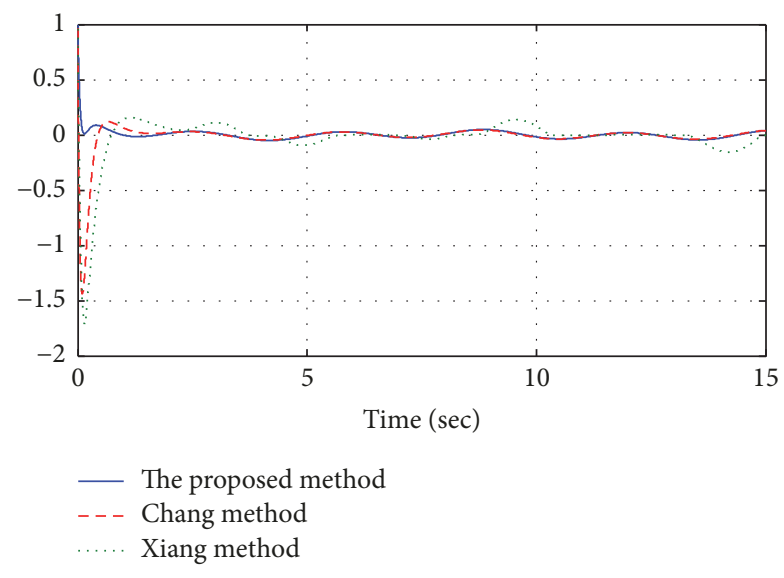

FIGURE 4: System output $y_{1}$ of the three methods.

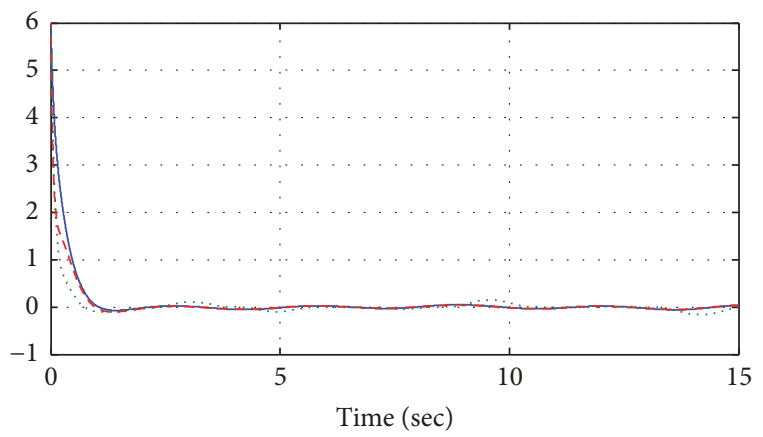

\footnotetext{
— The proposed method Chang method Xiang method
}

FIgURE 5: System output $y_{2}$ of the three methods.

The system states are finally bounded in a small region and robust stability of the closed-loop system is guaranteed. For comparison, the sliding surface and the control law,

$$
\begin{aligned}
\sigma(t) & =\left[\begin{array}{ll}
4.4132 & 1.3153
\end{array}\right] \mathbf{y}(t) \\
u(t) & =-2 \sigma(t)-4.5 \frac{\sigma(t)}{|\sigma(t)|+0.005}, \\
u(t) & =-\left[\begin{array}{ll}
4.6156 & 1.0774
\end{array}\right] \mathbf{y}(t) \\
- & \frac{1}{0.05}\left(\left[\begin{array}{ll}
0.5 & 0.5
\end{array}\right] \mathbf{y}(t)\right. \\
- & \left.\int_{0}^{t}\left[\begin{array}{ll}
4.6156 & 1.0774
\end{array}\right] \mathbf{y}(\tau) d \tau\right)
\end{aligned}
$$

proposed by Xiang et al. [6] and Chang et al. [4], are simultaneously simulated. The time responses of system outputs for the three cases are shown in Figures 4 and 5. Figure 6 shows the system input. Xiang et al. [6] developed an iterative LMI algorithm, which is relatively complex and hence not easy to implement, for reducing the high-gain control, while the proposed method uses the well-developed static output feedback technique to design the controller. As

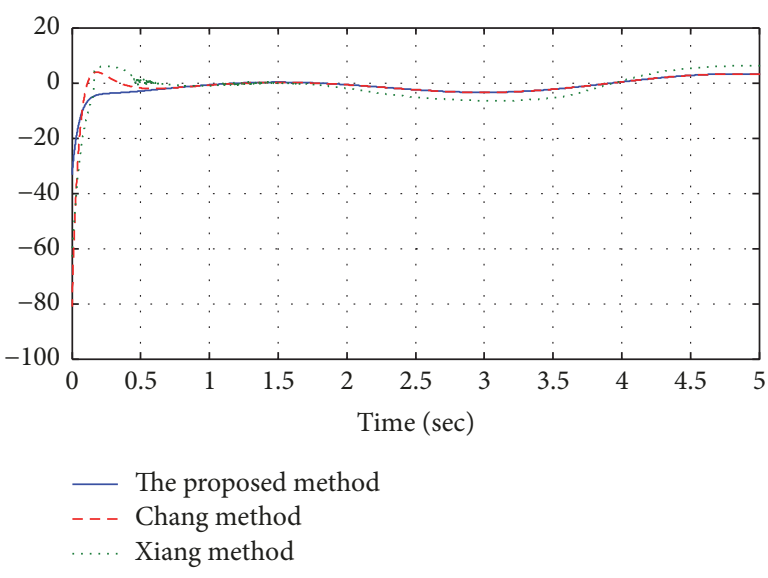

FIGURE 6: System input of the three methods.

seen from these figures, although the system involves the matched disturbance and the mismatched uncertain term, the property of disturbance attenuation is evident under the proposed algorithm and the high control effort is effectively reduced.

\section{Conclusions}

This study has proposed a design method of an output feedback control law for a linear MIMO system with mismatched uncertainty and disturbance. To fulfil the control saturation while achieving robustness simultaneously, the proposed control law can adjust robustness during the transient period and guarantee robust stability of the closed-loop system. When the control gain is increased, the equivalent input disturbance can be effectively eliminated, thus obtaining disturbance rejection. The simulation results have demonstrated that high performance could be obtained by applying the proposed scheme if certain tolerance of transient response is permitted.

\section{Conflicts of Interest}

The authors declare that there are no conflicts of interest regarding the publication of this paper.

\section{References}

[1] J. Gadewadikar, F. L. Lewis, K. Subbarao, K. Peng, and B. M. Chen, "H-infinity static output-feedback control for rotorcraft," Journal of Intelligent \& Robotic Systems, vol. 54, no. 4, pp. 629646, 2009.

[2] M. M. Arefi, J. Zarei, and H. R. Karimi, "Observer-based adaptive stabilization of a class of uncertain nonlinear systems," Systems Science \& Control Engineering, vol. 2, no. 1, pp. 362-367, 2014.

[3] J.-L. Chang and T.-C. Wu, "Disturbance observer based dynamic output feedback controller design for linear uncertain systems," Asian Journal of Control, vol. 15, no. 5, pp. 1261-1269, 2013. 
[4] J.-L. Chang, C.-P. Wu, and C.-L. Shih, "Output feedback high-gain proportional integral control for minimum phase uncertain systems," Journal of the Franklin Institute, vol. 352, no. 6, pp. 2314-2328, 2015.

[5] H. H. Choi, "Sliding-mode output feedback control design," IEEE Transactions on Industrial Electronics, vol. 55, no. 11, pp. 4047-4054, 2008.

[6] J. Xiang, W. Wei, and H. Su, "An ILMI approach to robust static output feedback sliding mode control," International Journal of Control, vol. 79, no. 8, pp. 959-967, 2006.

[7] J. M. Andrade-Da Silva, C. Edwards, and S. K. Spurgeon, "Sliding-mode output-feedback control based on LMIs for plants with mismatched uncertainties," IEEE Transactions on Industrial Electronics, vol. 56, no. 9, pp. 3675-3683, 2009.

[8] J.-L. Chang, "Dynamic output integral sliding-mode control with disturbance attenuation," Institute of Electrical and Electronics Engineers Transactions on Automatic Control, vol. 54, no. 11, pp. 2653-2658, 2009.

[9] J.-L. Chang, "Dynamic output feedback integral sliding mode control design for uncertain systems," International Journal of Robust and Nonlinear Control, vol. 22, no. 8, pp. 841-857, 2012.

[10] C. Edwards and S. K. Spurgeon, Sliding Mode Control Theory and Application, Taylor Francis, 1998.

[11] K. Ohnishi, M. Shibata, and T. Murakami, "Motion control for advanced mechatronics," IEEE/ASME Transactions on Mechatronics, vol. 1, no. 1, pp. 56-67, 1996.

[12] H. Shim and N. H. Jo, "An almost necessary and sufficient condition for robust stability of closed-loop systems with disturbance observer," Automatica, vol. 45, no. 1, pp. 296-299, 2009.

[13] Y. Zhang, L. Wang, J. Zhang, and J. Su, "Robust observer based disturbance rejection control for euler-lagrange systems," Mathematical Problems in Engineering, vol. 2016, Article ID 3839505, 2016.

[14] J.-H. She, M. Fang, Y. Ohyama, H. Hashimoto, and M. Wu, "Improving disturbance-rejection performance based on an equivalent-input-disturbance approach," IEEE Transactions on Industrial Electronics, vol. 55, no. 1, pp. 380-389, 2008.

[15] M. Corless and J. Tu, "State and input estimation for a class of uncertain systems," Automatica, vol. 34, no. 6, pp. 757-764, 1998.

[16] Q. P. Ha and H. Trinh, "State and input simultaneous estimation for a class of nonlinear systems," Automatica, vol. 34, no. 6, pp. 757-764, 1998.

[17] M. Darouach, M. Zasadzinski, and S. J. Xu, "Full-order observers for linear systems with unknown inputs," Institute of Electrical and Electronics Engineers Transactions on Automatic Control, vol. 39, no. 3, pp. 606-609, 1994.

[18] M. Hou and P. C. Muller, "Design of observers for linear systems with unknown inputs," Institute of Electrical and Electronics Engineers Transactions on Automatic Control, vol. 37, no. 6, pp. 871-875, 1992.

[19] B. Yao, M. Al-Majed, and M. Tomizuka, "High-performance robust motion control of machine tools: An adaptive robust control approach and comparative experiments," IEEE/ASME Transactions on Mechatronics, vol. 2, no. 2, pp. 63-76, 1997.

[20] A. Chakrabortty and M. Arcak, "Time-scale separation redesigns for stabilization and performance recovery of uncertain nonlinear systems," Automatica, vol. 45, no. 1, pp. 34-44, 2009.

[21] K.-K. D. Young, P. V. Kokotović, and V. I. Utkin, "A Singular Perturbation Analysis of High-Gain Feedback Systems," IEEE
Transactions on Automatic Control, vol. 22, no. 6, pp. 931-938, 1977.

[22] P. V. Kokotovic, H. K. Khalil, and J. O'Reilly, Singularly Perturbation Methods in Control: Analysis and Design, Academic Press, New York, NY, USA, 1986.

[23] H. K. Khalil, Nonlinear Systems, New Jersey, NJ, USA, PrenticeHall, 3rd edition, 2002. 


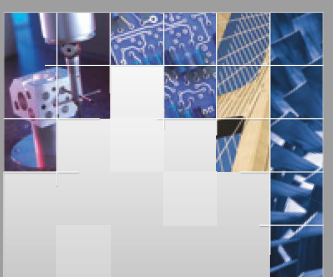

\section{Enfincering}
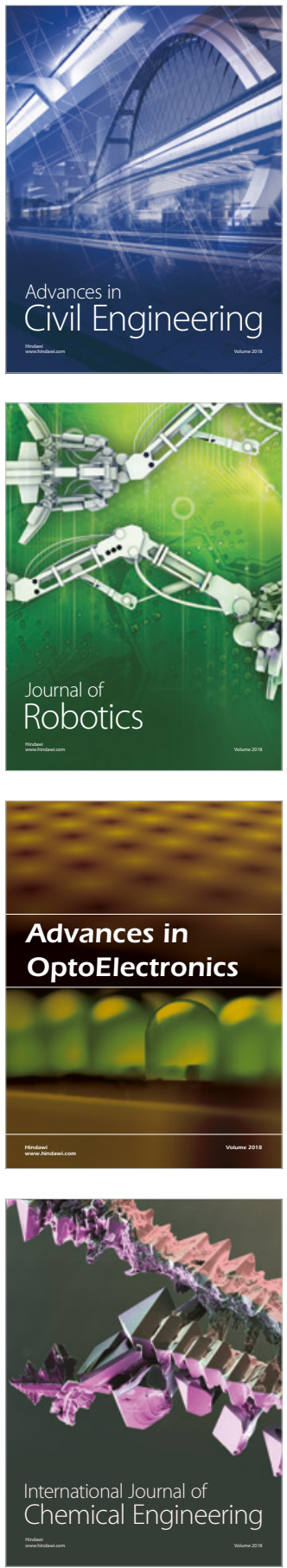

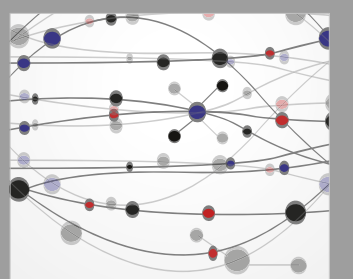

\section{Rotating \\ Machinery}

The Scientific World Journal

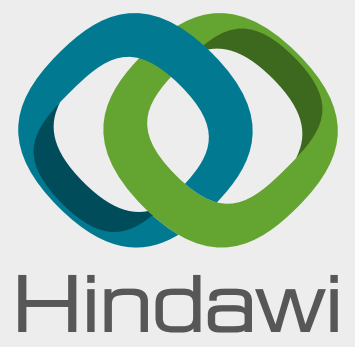

Submit your manuscripts at

www.hindawi.com
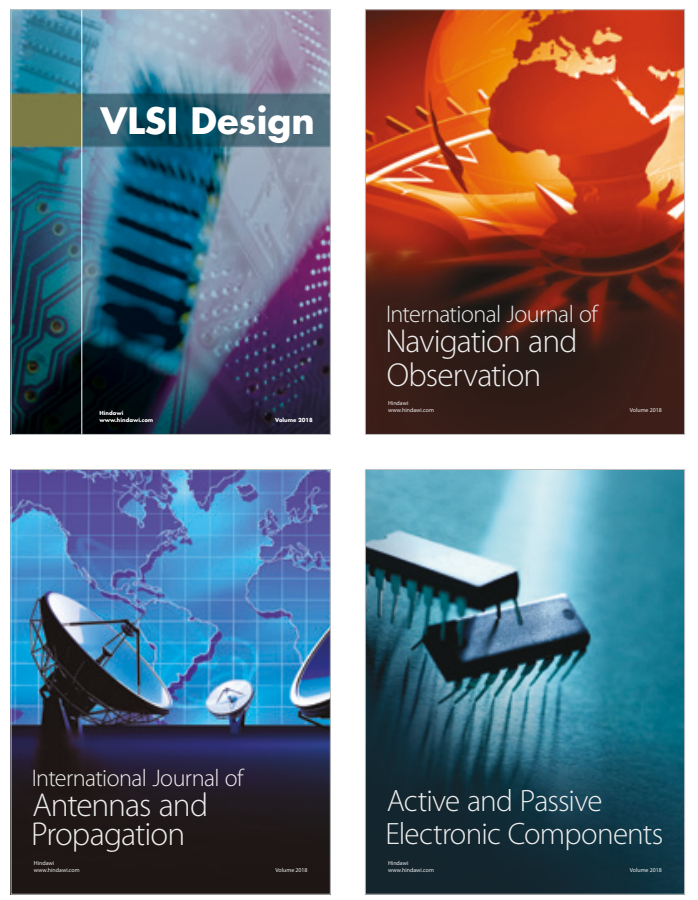
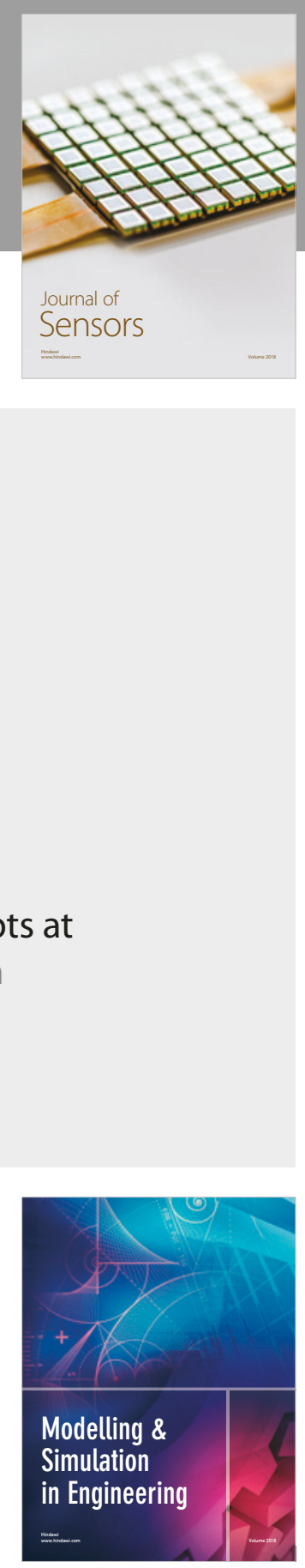

\section{Advances \\ Multimedia}
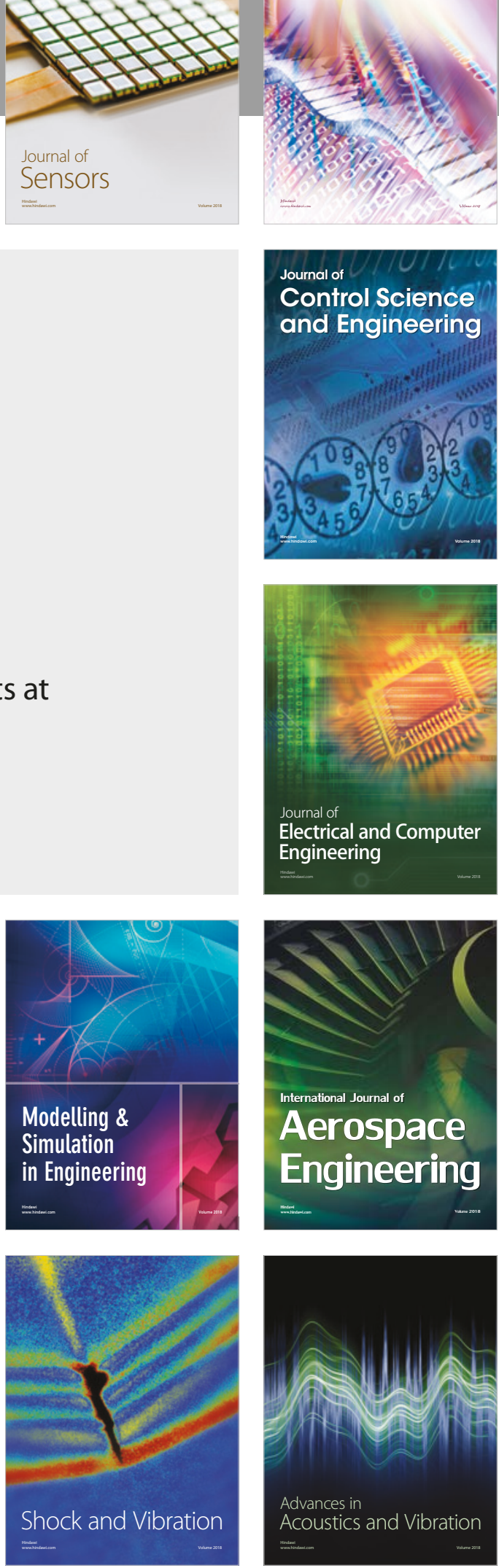